\section{MS7-05 Structure of the human 80S ribosome}

S. Kundhavai Natchiar ${ }^{1}$, Heena Khatter ${ }^{1}$, Alexander G Myasnikov $^{1}$, Bruno P Klaholz ${ }^{1}$

1. Centre for Integrative Biology (CBI), Department of Integrated Structural Biology, IGBMC (Institute of Genetics and of Molecular and Cellular Biology), 1 rue Laurent Fries, Illkirch, France

email: natchiar@igbmc.fr

Ribosomes are translational machineries in living cells that catalyse protein synthesis. Ribosome structures from various species are known to the atomic level, but that of the human ribosome has remained a challenge to address. We established a purification protocol to obtain homogenous $80 \mathrm{~S}$ ribosomes from $\mathrm{HeLa}$ cells and characterized them biophysically. Here we report the near-atomic structure of the human ribosome derived from high-resolution single particle cryo electron microscopy using the in-house Titan Krios electron microscope, advanced image processing and dedicated computing resources, and atomic model building using new crystallography refinement procedures. The structure has an average resolution of $3.6 \AA$ and reaches $2.9 \AA$ resolution in the most stable regions and thus provides unprecedented insights into rRNA entities and amino acid side-chains. The final atomic model comprises 220000 atoms across the 5866 nucleotide residues and $\sim 11590$ amino acids of the 80 proteins, the 4 rRNA's and E-site tRNA. The structure reveals the specific molecular recognition of the exit site tRNA with rRNA and protein elements of the ribosome. It highlights atomic details of the molecular interactions at the ribosomal subunit interface which is seen to strongly remodel upon rotational movements of the ribosomal subunits. Moreover, the structure paves the way for studying various diseases associated with deregulated protein synthesis.

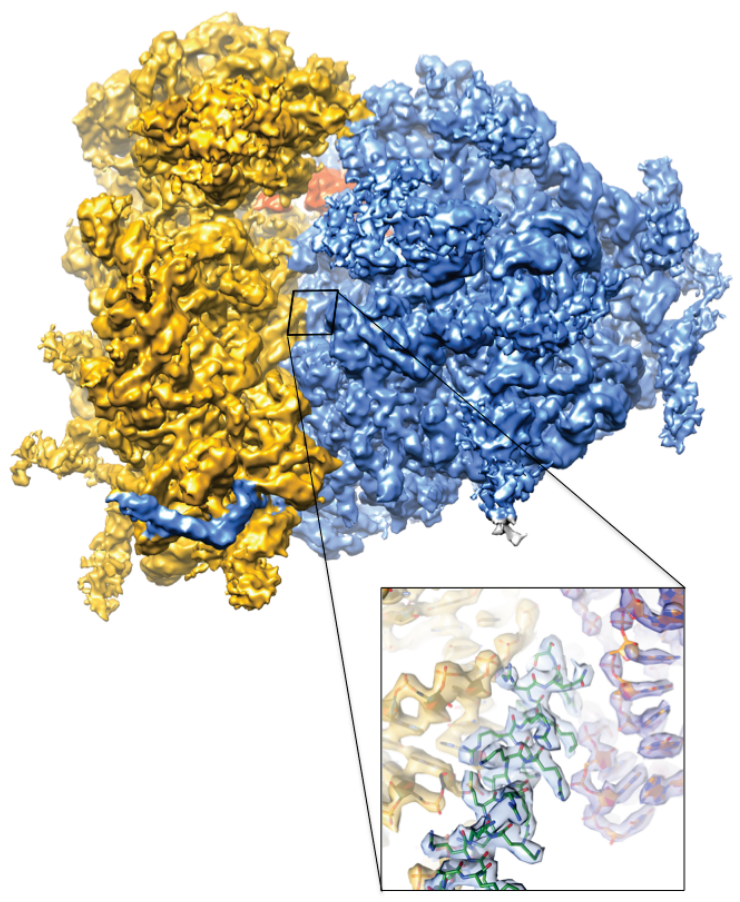

Figure 1. CryoEM towards atomic resolution

Keywords: Human $80 \mathrm{~S}$ ribosome, E-site tRNA, Cryo electron microscopy 